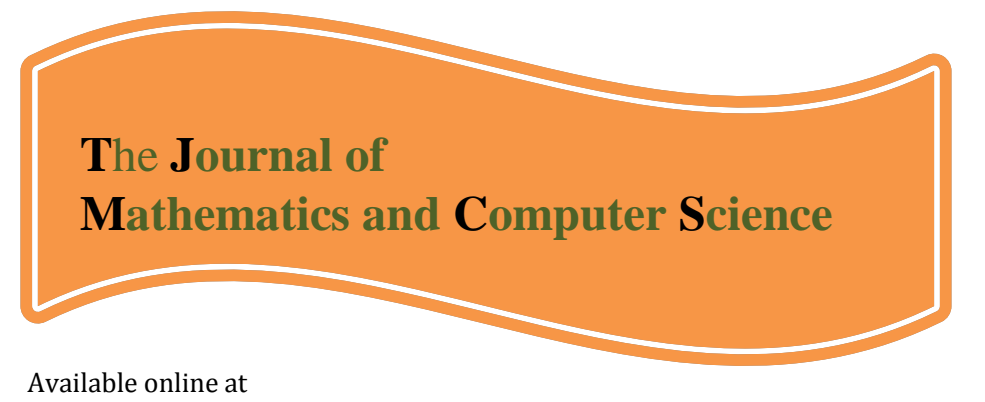

\title{
http://www.TJMCS.com
}

The Journal of Mathematics and Computer Science Vol .5 No.4 (2012) 331-336

\section{Analytical Solution of Two-Dimensional Viscous Flow between Slowly Expanding or Contracting Walls with Weak Permeability}

\author{
Arash Yahyazadeh \\ Department of Chemichal Engineering, University of Semnan, Semnan, Iran \\ sirarash65@yahoo.com \\ Hossein Yahyazadeh \\ Department of Mechanical Engineering, Babol University of Technology, Babol, Iran \\ Hossein_y62@yahoo.com \\ Mohammadtaghi Khalili \\ Department of Mechanical Engineering, Babol University of Technology, Babol, Iran \\ mt.e.khalili@gmail.com \\ Milad Malekzadeh \\ Department of Electrical and Computer Engineering, Babol Univiversity of Technology, Babol, Iran \\ m.malekzade@stu.nit.ac.ir
}

Received: February 2012, Revised: November 2012

Online Publication: December 2012

\begin{abstract}
In this article the problem of two-dimensional viscous flow between slowly expanding or contracting walls with weak permeability is presented and Homotopy Perturbation Method (HPM) is employed to compute an approximation to the solution of the system of nonlinear differential equations governing the problem. Comparisons are made between the Numerical solution (NM) and the results of the He's Homotopy Perturbation Method (HPM).
\end{abstract}

Keywords: Homotopy perturbation method; analytical solution; two dimension viscous flow.

\section{Mathematics Subject Classification: Primary 54A40; Secondary 46S40.}

\section{Introduction.}

The flow of Newtonian and non-Newtonian fluids in a porous surface channel has attracted the interest of many investigators in view of its applications in engineering practice, particularly in chemical industries. Examples of these are the cases of boundary layer control, transpiration cooling and gaseous diffusion. Theoretical research on steady flow of this type was initiated by 
Berman [1] who found a series solution for the two-dimensional laminar flow of a viscous incompressible fluid in a parallel-walled channel for the case of a very low cross-flow Reynolds number. After his work, this problem has been studied by many researchers considering various variations in the problem, e.g., Choi et al. [2] and references cited therein. For the case of a converging or diverging channel with a permeable wall, if the Reynolds number is large and if there is suction or injection at the walls whose magnitude is inversely proportional to the distance along the wall from the origin of the channel, a solution for laminar boundary layer equations can be obtained [3].

\section{A brief explanation on Homotopy Perturbation Method}

The Homotopy perturbation method (HPM) is well-known method to solve the nonlinear equations [4]. This method is introduced by He for the first time. This method has been used by many authors such as Ganji and the references therein to handle a wide variety of scientific and engineering applications such as linear and nonlinear, homogeneous and inhomogeneous as well, because these methods continuously deform a difficult problem into a simple one, which is easy to solve. They were shown by many authors that these methods provide improvements over existing numerical techniques. With the rapid development of nonlinear science, many different methods were proposed to solve various boundary-value problems (BVP) and fractional order, such as Homotopy perturbation method (HPM). These methods give successive approximations of high accuracy of the solution.

To explain this method, consider following function:

$A(u)+f(r)=0$

With the boundary condition of:

$B\left(u, \frac{\partial u}{\partial n}\right)=0$

Where $\mathrm{A}(\mathrm{u})$ is defined as follows:

$A(u)=L(u)+N(u)$

Homotopy perturbation structure is as the following equation:

$v(r, p)=\Omega \times[0,1] \rightarrow R H(v, p)=L(v)-L\left(u_{0}\right)+p L\left(u_{0}\right)+p[N(v)-f(r)]=0$

Obviously, from Esq. (2.4) we have:

$$
\begin{aligned}
& H(v, 0)=L(v)-L\left(u_{0}\right)=0 \\
& H(v, 1)=A(v)-f(r)=0
\end{aligned}
$$

Where, $\mathrm{p} \in[0,1]$ is an embedding parameter and $u_{o}$ is the first approximation that satisfies the boundary condition.

The process of changes in $\mathrm{p}$ from zero to unity is that of $v(r, p)$ changing from $u_{o}$ to $\mathrm{u}(\mathrm{r})$. We consider $v$ as following:

$v=v_{0}+P v_{1}+P^{2} v_{2}$

And the best approximation for equation answer is: 
$u=\operatorname{Lim} v=v_{0}+v_{1}+v_{2}+\ldots$

$$
P \rightarrow 1
$$

\section{Mathematical Formulation}

Consider the laminar, isothermal, and incompressible flow in a rectangular domain bounded by two permeable surfaces that enable the fluid to enter or exit during successive expansions or contractions $[6,7]$. One side of the cross section, representing the distance (2a) between the walls is taken to be smaller than the other two (W and L).Both walls are assumed to have equal permeability and to expand uniformly at a time dependent rate $a$. Furthermore, the origin $\hat{x}=0$ is assumed to be the center of the classic squeeze film problem. This enables us to assume flow symmetry about $\hat{x}=0$.Under these assumptions, the equations for continuity and motion become.

$\frac{\partial \hat{u}}{\partial \hat{x}}+\frac{\partial \hat{v}}{\partial \hat{y}}=0$

$\frac{\partial \hat{u}}{\partial t}+\hat{u} \frac{\partial \hat{u}}{\partial \hat{x}}+\hat{v} \frac{\partial \hat{v}}{\partial \hat{y}}=-\frac{1}{\rho} \frac{\partial \hat{\rho}}{\partial \hat{x}}+v \nabla^{2} \hat{u}$

$\frac{\partial \hat{v}}{\partial t}+\hat{u} \frac{\partial \hat{v}}{\partial \hat{x}}+\hat{v} \frac{\partial \hat{v}}{\partial \hat{y}}=-\frac{1}{\rho} \frac{\partial \hat{\rho}}{\partial \hat{y}}+v \nabla^{2} \hat{v}$

Where, $\hat{u}, \hat{v}, \hat{\rho}$ and $t$ are the dimensional pressure, density, kinematic viscosity, and time. Auxiliary conditions can be specified such as

$u(\hat{x}, 0)=0, \hat{v}(a)=-\hat{v}(w)=-a / c$

$\partial \hat{u} / \partial \hat{y}(\hat{x}, 0)=0, \hat{v}(0)=0, \hat{u}(0, \hat{y})=0$

$F^{\prime \prime \prime}+\alpha\left(y F^{\prime \prime \prime}+2 F^{\prime \prime}\right)+\operatorname{Re} F F^{\prime \prime \prime}-\operatorname{Re} F^{\prime} F^{\prime \prime}=0$

With the boundary conditions

$F^{\prime \prime}(0)=0, F(0)=0, F^{\prime}(1)=0, F(1)=1$

where a prime denotes differentiation with respect to ${ }^{y}$ : Note that Berman's well-known ODE can be viewed as a special case of with $\alpha=0$.

\section{Application of analytical solution methods to Two-Dimensional Viscous Flow}

According to HPM, we can construct Homotopy of equation as follows:

$$
\begin{aligned}
& (1-P)\left[F^{(4)}(y)\right]+p\left[F^{(4)}(y)+\alpha\left(y F^{\prime \prime \prime}(y)+2 F^{\prime \prime}(y)\right)+\operatorname{Re} F(y) F^{\prime \prime \prime}(y)-\operatorname{Re} F^{\prime}(y) F^{\prime \prime}(y)\right]=0 \\
& F(y)=f_{0}(y)+p f_{1}(y)+p^{2} f_{2}(y)+p^{3} f_{3}(y)+p^{4} f_{4}(y)+p^{5} f_{5}(y) \\
& +p^{6} f_{6}(y)+p^{7} f_{7}(y)+p^{8} f_{8}(y)+p^{9} f_{9}(y)+p^{10} f_{10}(y)
\end{aligned}
$$

By substituting $F$ from Eq.(3.2) into Eq.(3.1) and after some simplifications and rearranging based on powers of p-terms. we have:

Obtained equation

$$
p^{0}=0 \rightarrow f_{0}^{(4)}(y)=0
$$


boundary condition

$$
f_{0}(0)=0 \quad f_{0}^{\prime \prime}(0)=0 \quad f_{0}^{\prime}(1)=0 \quad f_{0}(1)=1
$$

The solution of Eq.(4.1.3) and with (4.1.4),is obtained $f_{0}(\mathrm{y})$

$$
f_{0}(y)=-0.5 y^{3}+1.5 y
$$

In the same manner

$$
p^{1} \rightarrow f_{1}^{(4)}(y)-f_{0}^{\prime}(y) f_{0}^{\prime \prime}(y)+f_{0}^{\prime \prime}(y)+0.5 y f_{0}^{\prime \prime \prime}(y)+f_{0}(y) f_{0}^{\prime \prime \prime}(y)=0
$$

$$
\begin{aligned}
& f_{1}(0)=0, f_{1}^{\prime \prime}(0)=0, f_{1}^{\prime}(1)=0, f_{1}(1)=0 \\
& f_{1}(y)=\frac{1}{280} y^{7}+\frac{3}{80} y^{5}-\frac{3}{35} y^{3}+\frac{5}{112} y \\
& p^{2} \rightarrow f_{2}^{(4)}(y)+f_{1}(y) f_{0}^{\prime \prime \prime}(y)-f_{1}^{\prime}(y) f_{0}^{\prime \prime}(y)+f_{1}^{\prime \prime}(y)-f_{0}^{\prime}(y) f_{1}^{\prime \prime}(y)+0.5 y f_{1}^{\prime \prime \prime}(y)+f_{0}(y) f_{1}^{\prime \prime \prime}(y)=0
\end{aligned}
$$

$$
\begin{aligned}
& f_{2}(0)=0, f_{2}^{\prime \prime}(0)=0, f_{2}^{\prime}(1)=0, \quad f_{2}(1)=0 \\
& f_{2}(y)=\frac{1}{92400} y^{11}-\frac{5}{8064} y^{9}-\frac{21674999999}{5880000000000} y^{7} \\
& +\frac{53999999999}{8400000000000} y^{5}+\frac{76109374967}{121275000000000} y^{3}-\frac{1785624999857}{646800000000000} y
\end{aligned}
$$

$f_{3}, f_{4}(y) \ldots$ were too long to mentioned here, therefore they are shown graphically.

\section{Result and Discussion}

In this section, we compare the results of analytical solutions. Figure (1) exhibits the approximate solutions of the equation for $\operatorname{Re}=1$ and $\alpha=0$ using HPM, ADM, VIM. Figure (2) exhibits the approximate solutions for $\operatorname{Re}=1$ and $\alpha=-0.5$ using HPM, ADM, VIM. Figure (3) exhibits the approximate solutions of the proposed equation for $\operatorname{Re}=5$ and $\alpha=-0.5$ using HPM, ADM, VIM. Figure (4) exhibits the approximate solutions for $\operatorname{Re}=7$ and $\alpha=-0.5$ using HPM, ADM, and VIM. It is obvious through these figures that HPM was capable of solving them with successive rapidly convergent approximations.

\section{CONCLUSIONS}

Results clearly show that HPM which was applied to the two-dimensional viscous flow between slowly expanding or contracting walls with weak permeability problem, were capable of solving them with successive rapidly convergent approximations without any restrictive assumptions or transformations causing changes in the physical definition of the problem. The results show that this scheme provides excellent approximations to the solution of this nonlinear equation with high accuracy. Finally, it has been attempted to show the capabilities and wide-range applications of the 
Homotopy Perturbation Method (HPM) in comparison with the Variational Iteration Method (VIM), Adomian Decomposition Method (ADM) and numerical solution of two-dimensional viscous flow between slowly expanding or contracting walls with weak permeability problems.

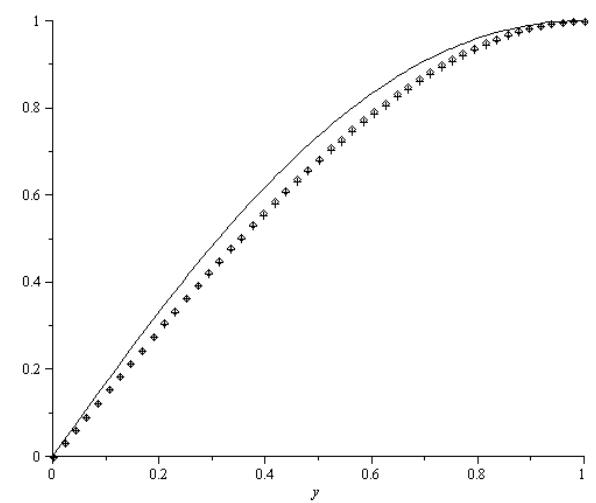

Figure1: comparison of result of analytical solutions For $\alpha=0.5, \operatorname{Re}=1$, HPM:-,ADM: - ,VIM: ?

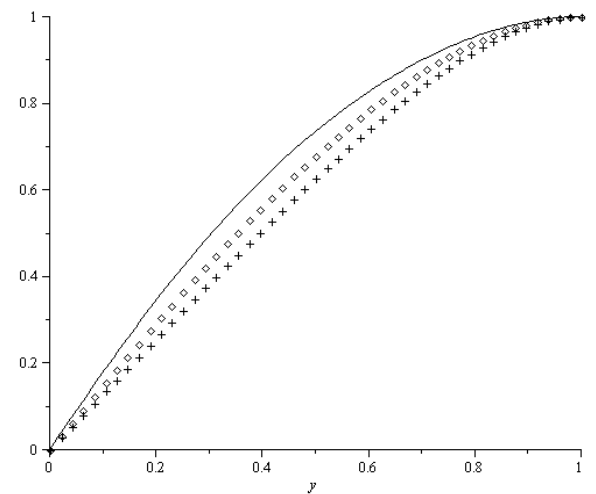

Figure3: comparison of result of analytical solutions For $\alpha=-0.5, \operatorname{Re}=5$, HPM:-, ADM: -, VIM:?

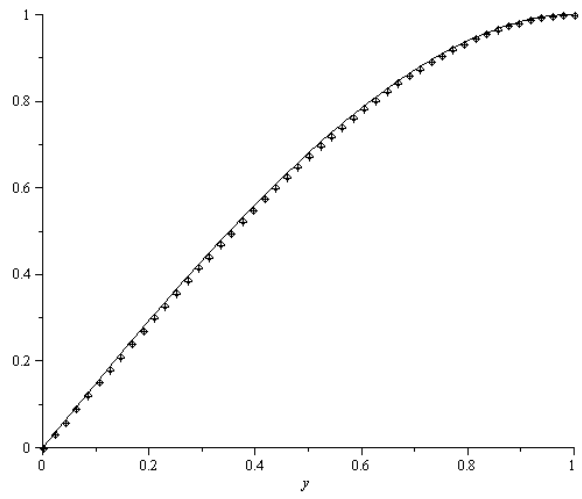

Figure2: comparison of result of analytical solutions For $\alpha=-0.5, \operatorname{Re}=1$, HPM, ADM: -, VIM: ?

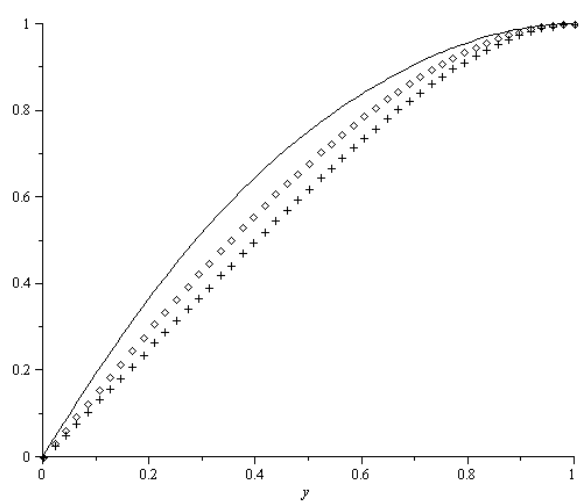

Figure4: comparison of result of analytical solutions For $\alpha=-0.5, \operatorname{Re}=7$, HPM:-, ADM: -, VIM:

Table 1: Comparison of results of different analytical methods for $\operatorname{Re}=1 \alpha=0.5$

\begin{tabular}{|c|c|c|c|}
\hline y & ADM & HPM & VIM \\
\hline $\mathbf{0}$ & 0 & 0 & 0 \\
\hline 0.05 & 0.074217 & 0.077016 & 0.074347 \\
\hline 0.1 & 0.148112 & 0.153594 & 0.148271 \\
\hline 0.15 & 0.221352 & 0.229297 & 0.221373 \\
\hline 0.2 & 0.29359 & 0.303693 & 0.293278 \\
\hline 0.25 & 0.364472 & 0.376352 & 0.363634 \\
\hline 0.3 & 0.43363 & 0.446855 & 0.432102 \\
\hline 0.35 & 0.500687 & 0.514786 & 0.498354 \\
\hline 0.4 & 0.565257 & 0.579743 & 0.562071 \\
\hline 0.45 & 0.626944 & 0.641332 & 0.622932 \\
\hline 0.5 & 0.685346 & 0.699174 & 0.680616 \\
\hline 0.55 & 0.740057 & 0.752904 & 0.734793 \\
\hline 0.6 & 0.790667 & 0.802172 & 0.785121 \\
\hline 0.65 & 0.836766 & 0.846645 & 0.83124 \\
\hline 0.7 & 0.877944 & 0.88601 & 0.872767 \\
\hline 0.75 & 0.9138 & 0.919972 & 0.909293 \\
\hline 0.8 & 0.94394 & 0.948259 & 0.940377 \\
\hline 0.85 & 0.967982 & 0.97062 & 0.965538 \\
\hline 0.9 & 0.985564 & 0.986829 & 0.984254 \\
\hline 0.95 & 0.996342 & 0.996681 & 0.995951 \\
\hline 1 & 1 & 1 & 1 \\
\hline
\end{tabular}


A. Yahyazadeh, H. Yahyazadeh, M. Khalili, M. Malekzadeh / TJMCS Vol .5 No.4 (2012) 331-336

\section{References:}

[1] Berman, A.S. Laminar, Flow in Channels with Porous Walls, J. Appl. Phys., 24 (1953) 1232-1235.

[2] J.J. Choi, Z. Rusak and J.A. Tichy, Maxwell Fluid Suction Flow in a Channel, J. Non- Newtonian Fluid Mech., 85 (1999) 165-187.

[3] L. Rosenhead, Laminar Boundary Layers, Oxford: Clerendon Press, (1963) 250-251.

[4] D.D. Ganji, A. Rajabi, ASSESSMENT OF HOMOTOPY PERTURBATION AND PERTURBATION METHODS IN HEAT RADIATION EQUATIONS.

[5] Cihat Arslanturk, A decomposition method for fin efficiency of convective straight fins with temperature-dependent thermal conductivity, International Communications in Heat and Mass Transfer 32 (2005) 831-841

[6] J. Majdalani, C. Zhou, C. A. Dawson, Two-dimensional viscous flow between slowly expanding or contracting walls with weak permeability, Journal of Biomechanics, 35 (2002) 1399-1403.

[7] Z. Z. Ganji, D. D. Ganji, A. Janalizadeh, ANALYTICAL SOLUTION OF TWO-DIMENSIONAL VISCOUS FLOW BETWEEN SLOWLY EXPANDING OR CONTRACTING WALLS WITH WEAK PERMEABILITY, Mathematical and Computational Applications, Vol. 15, No. 5, pp. 957-961, 2010. 